\title{
CONFORMALLY FLAT RIEMANNIAN MANIFOLDS ADMITTING A ONE-PARAMETER GROUP OF CONFORMAL TRANSFORMATIONS
}

\author{
MORIO OBATA
}

\section{Introduction}

It is a classical theorem of H. A. Schwarz [4] that a compact Riemann surface of genus greater than 1 has only a finite number of conformal transformations. It is furthermore known that in the case of genus 1 , i.e. on a torus, there is no one-parameter group of non-isometric conformal transformations.

For Riemannian manifolds the following is a well-known conjecture:

Conjecture. If a compact Riemannian n-manifold $M, n>2$, admits an essential one-parameter group of conformal transformations, then $M$ is conformorphic to a Euclidean sphere $S^{n}$.

Here a one-parameter group and the vector field defined by it are said to be essential if there is no Riemannian metric, conformal to the original one, with respect to which the group is a group of isometries, and by "conformorphic" we mean "conformally diffeomorphic". Furthermore, throughout this paper manifolds under consideration are assumed to be connected and of $C^{\infty}$.

In a previous paper [3], we have proved the following Theorems A and B:

Theorem A. Let $M$ be a Riemannian n-manifold, $n>2$, admitting an essential one-parameter graup $f_{t}$ of conformal transformations. Then

(i) $f_{t}$ has a fixed point,

(ii) $M$ is conformorphic to either a Euclidean $n$-sphere $S^{n}$ or a oncepunctured $n$-sphere $S^{n}-\left\{p_{\infty}\right\}$ provided that the vector field defined by $f_{t}$ has nonvanishing divergence at each of the fixed points of $f_{t}$.

The vector field induced by a one-parameter group of conformal transformations is called a conformal vector field, and a fixed point of a one-parameter group is called a zero or a singular point of the corresponding vector field.

Theorem B. Let $u$ be an essential conformal vector field on a Euclidean $n$-sphere $S^{n}$. Then u satisfies one of the following two properties:

(i) $u$ has exactly one singular point $p_{0}$, at which the divergence of $u$ vanishes, and the orbit $f_{t}(p)$ of $u$ through a point $p$ satisfies

$$
\lim _{t \rightarrow \pm \infty} f_{t}(p)=p_{0}
$$

Received March 18, 1969. Supported by an NSF Senior Foreign Scientist Fellowship at Lehigh University in 1968-1969. 
(ii) $u$ has exactly two singular points $p_{0}$ and $p_{\infty}$, at each of which the divergence is not zero, and the orbit $f_{t}(p)$ through a point $p \notin\left\{p_{0}, p_{\infty}\right\}$ connects $p_{0}$ and $p_{\infty}$.

The vector field in Theorem A corresponds to a vector field of the second type in Theorem B, and the conformal-flatness of a Riemannian $n$-manifold, $n>2$, is an implication of the existence of such a vector field.

The purpose of the present paper is to establish the

Theorem. Let $M$ be a conformally flat Riemannian $n$-manifold, $n>2$, with finite fundamental group. If $M$ admits an essential one-parameter group $f_{t}$ of conformal transformations, then $M$ is conformorphic to either a Euclidean $n$-sphere $S^{n}$ or a once-punctured Euclidean $n$-sphere $S^{n}-\left\{p_{\infty}\right\}$.

This is a parital solution to the conjecture and a generalization of a result of Nagano [2] for the transitive group of conformal transformations.

\section{Lemmas}

Lemma 1. Let $G$ and $\Gamma$ be respectively groups of isometries and conformal transformations of a Riemannian manifold $(M, g)$. If $G$ and $\Gamma$ are commutative element-wise, and $\Gamma$ is compact, then there is a Riemannian metric $g^{*}$ conformal to $g$ such that both $G$ and $\Gamma$ are groups of isometries of $\left(M, g^{*}\right)$.

Proof. We put

$$
g^{*}=\int_{\Gamma} \gamma^{*} g d \gamma, \quad \gamma \in \Gamma,
$$

where $d \gamma$ denotes the invariant measure on $\Gamma$ with $\int_{\Gamma} d \gamma=1$. Then $g^{*}$ is a Riemannian metric conformal to $g$ since each $\gamma \in \Gamma$ is a conformal transformation. Obviously $\Gamma$ is a group of isometries of $\left(M, g^{*}\right)$. For any $\sigma \in G$, we have

$$
\sigma^{*} g^{*}=\int_{\Gamma} \sigma^{*} \gamma^{*} g d \gamma=\int_{\Gamma} \gamma^{*} \sigma^{*} g d \gamma=\int_{\Gamma} \gamma^{*} g d \gamma=g^{*} .
$$

Thus $G$ is a group of isometries of $\left(M, g^{*}\right)$

Lemma 2. Let $G$ be an essential one-parameter group of conformal transformations of a Riemannian manifold $(M, g)$. If the fundamental group $\Gamma$ of $M$ is finite, then $G$ is essential as a group of conformal transformations of the universal covering space $(\bar{M}, \bar{g})$ of $(M, g)$, where $\bar{g}$ is locally isometric to $g$ by means of the natural projection.

Proof. Since $(\bar{M}, \bar{g})$ is the Riemannian covering of $(M, g), \Gamma$ is a finite group of isometries. $G$ acts on $(\bar{M}, \bar{g})$ as a group of conformal transformations and $G$ and $\Gamma$ are commutative element-wise. 
Assume that $G$ is inessential, so that on $\bar{M}$ there is a Riemannian metric $\bar{h}$ conformal to $\bar{g}$ such that $G$ is a group of isometries with respect to $\bar{h}$. Obviously $\Gamma$ is a group of conformal transformations of $(\bar{M}, \bar{h})$. Since $\Gamma$ is finite and commutative with $G$ element-wise, by Lemma 1 , on $\bar{M}$ there is a Riemannian metric $\bar{h}^{*}$ conformal to $\bar{h}$ and hence to $\bar{g}$, such that both $\Gamma$ and $G$ are groups of isometries of $\left(M, \bar{h}^{*}\right)$. Since $\Gamma$ is a group of isometries, $\bar{h}^{*}$ can be projected to a Riemannian metric $h$ on $M$ so that $\pi^{*} h=h^{*}$, where $\pi$ denotes the natural projection $\bar{M} \rightarrow M$. Therefore $G$ is a group of isometries of $(M, h)$, contrary to our assumption that $G$ is essential on $(M, g)$.

\section{Proof of the theorem}

Let $(\bar{M}, \bar{g})$ be the Riemannian universal covering space of $(M, g)$. Then $f_{t}$ acts on $(\tilde{M}, \bar{g})$ as a group of conformal transformations and is essential by Lemma 2 . Let $\tilde{u}$ be the corresponding conformal vector field on $(\bar{M}, \bar{g})$. Clearly $\pi \tilde{u}=u$. Since $(\bar{M}, \bar{g})$ is conformally flat and simply connected, it is conformorphic to an open set $W$ of $S^{n}[1]$, and by this conformorphism, $\tilde{u}$ corresponds to a vector field $U$ on $W$. Let $\tilde{p}_{0}$ be a singular point of $\tilde{u}$, and $\tilde{P}_{0}$ the corresponding singular point of $U$. Since $U$ is uniquely determined by the values of $U$, the covariant derivatives $\nabla U$ of $U$, and the divergence $\Phi$ of $U$, at $P_{0}$, [2], $U$ can be extended to a conformal vector field $\bar{U}$ on $S^{n}$. It is clear that $\tilde{U}$ is essential on $S^{n}$. Let $\bar{f}_{t}$ be the one-parameter group generated by $\bar{U}$. Then $W$ must be invariant by $\bar{f}_{t}$ so that $\bar{f}_{t}(W) \subset W$.

i) If $U$ has vanishing divergence at $P_{0}$, then $P_{0}$ is the only singular point of $\widehat{U}$, and by Theorem B there is no invariant open subset of $S^{n}$ except $S^{n}$ itself. Therefore $W=S^{n}$, and $(\bar{M}, \bar{g})$ is conformorphic to $S^{n}$.

Let $p_{0}=\pi\left(\tilde{p}_{0}\right)$. Then any points $\tilde{p} \in \pi^{-1}\left(p_{0}\right)$ are singular points of $\tilde{u}$ on $\bar{M}$. However $\tilde{u}$ has only one singular point and therefore the fundamental group is trivial. Hence $(M, g)$ itself is conformorphic to $S^{n}$.

ii) If $U$ has nonvanishing divergence at $p_{0}$, then on account of i) the divergence never vanishes at any other singular points of $\tilde{U}$, if any. Therefore $u$ has the same property as $U$, and by Theorem $\mathrm{A},(M, g)$ is conformorphic to either $S^{n}$ or $S^{n}-\left\{p_{\infty}\right\}$.

\section{References}

[1] N. H. Kuiper, On conformally-flat spaces in the large, Ann. of Math. 50 (1949) 916-924.

[2] T. Nagano, On conformal transformations of Riemannian spaces, J. Math. Soc. Japan 10 (1958) 79-93

[3] M. Obata, Conformal transformations of Riemannian manifolds, J. Differential Geometry 4 (1970) 311-333.

[4] H. A. Schwarz, Gesammelte Mathematische Abhandlungen, Springer, Berlin, 1890.

TOKYo Metropolitan University LEHIGH UNIVERSITY 
